\title{
Modified lattice Boltzmann model for axisymmetric flows
}

\author{
T. Reis and T. N. Phillips \\ School of Mathematics, Cardiff University, Cardiff, CF24 4AG, United Kingdom
}

(Received 5 January 2007; published 9 May 2007)

\begin{abstract}
A modified lattice Boltzmann model based on the two-dimensional, nine-velocity lattice-Bhatnagar-GrossKrook fluid is presented for axisymmetric flows. A spatially and temporally varying source term is incorporated into the evolution equation for the momentum distribution function on a two-dimensional Cartesian lattice. The precise form of the source term is derived through a Chapman-Enskog analysis, so that the additional axisymmetric contributions in the Navier-Stokes equations are furnished when written in the cylindrical polar coordinate system.
\end{abstract}

DOI: $10.1103 /$ PhysRevE.75.056703

\section{INTRODUCTION}

The formulation of the standard lattice Boltzmann model for predicting the flow of incompressible fluids is based on the Cartesian coordinate system. However, numerous important flow problems exist for which there is axial symmetry, e.g., flow past a cylinder or sphere in a confined channel. The computational demand required for three-dimensional (3D) lattice Boltzmann models (LBMs) is considerably greater than for the 2D case. Therefore an axisymmetric LBM, which will depend on only two coordinates, is highly desirable, since it makes computational sense to take advantage of any reduction in dimension that can be accrued from geometrical considerations. Alternatively, a reduction in dimensionality also allows for greater spatial refinement through the availability of additional degrees of freedom that would have been required in the third dimension.

Halliday et al. [1] demonstrated how the evolution equation for the momentum distribution function within a $2 \mathrm{D}$ Cartesian framework may be adjusted by adding suitable source terms in order to recover the axisymmetric NavierStokes equations in the macroscopic limit. The first- and second-order terms in an expansion of the source term are chosen so that the terms in the lattice continuity and momentum equations, respectively, arising from the cylindrical polar coordinate system are recovered. Premnath and Abraham [2] adopted a similar approach for multiphase flows by including temporally and spatially dependent source terms to account for the axisymmetric contributions of the order parameter of the fluid phases and inertial, viscous, and surface tension forces.

In the present paper we follow the general philosophy embodied in the paper of Halliday et al. [1] but perform the Chapman-Enskog analysis in an alternative manner. The second-order equation that results from the general analysis performed in this paper differs from the one derived by Halliday et al. [1] in that it contains fewer terms. Furthermore, the form of the source terms that are derived allows for a more efficient implementation of the LBM for axisymmetric flow problems due to the reduction in the number of terms that require numerical differentiation.

\section{GOVERNING EQUATIONS IN AXISYMMETRIC GEOMETRIES}

Consider the flow of an incompressible, isotropic fluid through a three-dimensional pipe. Let $\mathbf{e}_{r}, \mathbf{e}_{\theta}$, and $\mathbf{e}_{z}$ be the
PACS number(s): 47.11.- $\mathrm{j}$

standard orthonormal unit vectors defining a cylindrical coordinate system:

$$
\mathbf{e}_{r}=\left(\frac{x}{r}, \frac{y}{r}, 0\right), \quad \mathbf{e}_{\theta}=\left(\frac{y}{r},-\frac{x}{r}, 0\right), \quad \mathbf{e}_{z}=(0,0,1),
$$

where $r=\sqrt{x^{2}+y^{2}}, x=r \cos \theta$, and $y=r \sin \theta$. If the solution to the Navier-Stokes equation is of the form

$$
\mathbf{u}=u_{r}(r, z) \mathbf{e}_{r}+u_{z}(r, z) \mathbf{e}_{z},
$$

that is, the velocity field does not depend on $\theta$, then the flow is said to be axisymmetric (without swirl). The continuity equation in cylindrical coordinates is

$$
\frac{\partial u_{r}}{\partial r}+\frac{u_{r}}{r}+\frac{\partial u_{z}}{\partial z}=0,
$$

and the components of the momentum equation are

$$
\begin{gathered}
\frac{\partial u_{r}}{\partial t}+u_{r} \frac{\partial u_{r}}{\partial r}+u_{z} \frac{\partial u_{r}}{\partial z}=-\frac{1}{\rho} \frac{\partial P}{\partial r}+\nu\left(\frac{\partial^{2} u_{r}}{\partial r^{2}}+\frac{1}{r} \frac{\partial u_{r}}{\partial r}-\frac{u_{r}}{r^{2}}+\frac{\partial^{2} u_{r}}{\partial z^{2}}\right) \\
\frac{\partial u_{z}}{\partial t}+u_{r} \frac{\partial u_{z}}{\partial r}+u_{z} \frac{\partial u_{z}}{\partial z}=-\frac{1}{\rho} \frac{\partial P}{\partial z}+\nu\left(\frac{\partial^{2} u_{z}}{\partial r^{2}}+\frac{1}{r} \frac{\partial u_{z}}{\partial r}+\frac{\partial^{2} u_{z}}{\partial z^{2}}\right)
\end{gathered}
$$

where $\nu$ is the kinematic viscosity.

By performing the following coordinate transformation:

$$
\begin{gathered}
(r, z) \mapsto(y, x), \\
\left(u_{r}, u_{z}\right) \mapsto\left(u_{y}, u_{x}\right),
\end{gathered}
$$

Eqs. (3)-(5) can be written in Cartesian-like coordinates:

$$
\begin{gathered}
\partial_{\alpha} u_{\alpha}=-\frac{u_{y}}{y}, \\
\frac{D \rho u_{\alpha}}{D t}+\partial_{\alpha} P-\nu \rho \nabla^{2} u_{\alpha}=\frac{\nu \rho}{y} \partial_{y} u_{\alpha}-\frac{\nu \rho u_{\alpha}}{y^{2}} \delta_{\alpha y},
\end{gathered}
$$

where $D / D t$ is the material derivative, $\delta_{\alpha \beta}$ is the Kronecker delta function, and $\alpha=x, y$. The terms on the right-hand side of the momentum equation (9) are the additional axisymmetric contributions that the source terms need to recover. 


\section{AXISYMMETRIC LATTICE BOLTZMANN EQUATION}

The lattice Boltzmann model is expressed in terms of an average population density $N_{i}$, and is based on Boltzmann's approximation that particles entering a collision are uncorrelated. This approximation enables a closed equation for the $N_{i}$ 's to be derived. Assuming that the space and time scales of the density and momentum fluctuations are large so that $N_{i}$ varies slowly in space and time, then the Chapman-Enskog expansion may be applied to the distribution function $N_{i}$ :

$$
N_{i}=N_{i}^{(0)}+\epsilon N_{i}^{(1)}+\epsilon^{2} N_{i}^{(2)}+\cdots,
$$

where $N_{i}^{(0)}$ is a local equilibrium distribution function and the perturbation parameter $\epsilon$ is analogous to the Knudsen number. The particular form of the equilibrium function for the two-dimensional, nine-velocity (D2Q9) lattice is due to Qian et al. [3]

$$
N_{i}^{(0)}=\rho W_{i}\left(1+3 \mathbf{c}_{i} \cdot \mathbf{u}-\frac{3}{2} u^{2}+\frac{9}{2}\left(\mathbf{c}_{i} \cdot \mathbf{u}\right)^{2}\right),
$$

where the weights $W_{i}$ are given by

$$
W_{i}= \begin{cases}\frac{4}{9}, & i=0, \\ \frac{1}{9}, & i=1,2,3,4, \\ \frac{1}{36}, & i=5,6,7,8 .\end{cases}
$$

The kinetic and hydrodynamic modes of the flow are controlled by the spectral properties of the collision matrix, the elements of which may be regarded as a set of free parameters that may be chosen freely, subject to the conservation constraints, to obtain the desired macroscopic quantities [4]. Since transport is related to just one nonzero eigenvalue of the collision matrix, the evolution equation can be simplified to yield the lattice-Bhatnagar-Gross-Krook (LBGK) evolution equation [3]:

$$
N_{i}\left(\mathbf{x}+\mathbf{c}_{i}, t+1\right)=N_{i}(\mathbf{x}, t)-\omega\left(N_{i}-N_{i}^{(0)}\right),
$$

where $\omega$ is a relaxation parameter in the range $0<\omega<2$.

The density $\rho$ and momentum $\rho \mathbf{u}$ are defined by the zeroth and first moments of the population density, respectively:

$$
\begin{gathered}
\rho=\sum_{i} N_{i}, \\
\rho \mathbf{u}=\sum_{i} N_{i} \mathbf{c}_{i} .
\end{gathered}
$$

The hydrodynamic equations of motion are obtained via a multiscale analysis in which the spatial and temporal differential operators are expanded about $\epsilon$. If the fluid density is assumed constant, a Taylor and Chapman-Enskog expansion of Eq. (13) recovers the incompressible Navier-Stokes equations.

To apply the lattice Boltzmann equation (LBE) to a range of flow problems, an internal or external force term may need to be added to Eq. (13). The exact form of this term depends on the mechanics in question, for example particlefluid suspensions [5], multiphase flows [6-8], viscoelastic fluids [9], or flow in an axisymmetric geometry [1]. A general representation of forcing terms within the LBE framework that considers discrete lattice effects has been proposed by Guo et al. [10]. With the intention of deriving Eqs. (8) and (9), a spatially and temporally varying microscopic term $S_{i}(\mathbf{x}, t)$ is introduced into the D2Q9 lattice Boltzmann equation $[1,2]$ :

$$
N_{i}\left(\mathbf{x}+\mathbf{c}_{i}, t+1\right)=N_{i}(\mathbf{x}, t)+\omega\left[N_{i}^{(0)}(\mathbf{x}, t)-N_{i}(\mathbf{x}, t)\right]+S_{i}(\mathbf{x}, t),
$$

and we take this source term to be at least $O(\epsilon)$ :

$$
S_{i}=\epsilon S_{i}^{(1)}+\epsilon^{2} S_{i}^{(2)}+\cdots,
$$

that is, there is no equilibrium term $S_{i}^{(0)}$.

The aim now is to adopt the strategy of Halliday et al. [1] and perform a Taylor and Chapman-Enskog expansion on Eq. (16) so that $S_{i}$ can be chosen in such a way as to recover Eqs. (3)-(5). To first order in $\epsilon$ we obtain

$$
\partial_{t_{1}} N_{i}^{(0)}+c_{i \alpha} \partial_{\alpha} N_{i}^{(0)}=-\omega N_{i}^{(1)}+S_{i}^{(1)},
$$

and the mass and momentum constraints yield

$$
\begin{gathered}
\partial_{t_{1}} \rho+\partial_{\alpha} \rho u_{\alpha}=\sum_{i=0}^{8} S_{i}^{(1)}, \\
\partial_{t_{1}} \rho u_{\alpha}+\partial_{\beta} \Pi_{\alpha \beta}=\sum_{i=0}^{8} S_{i}^{(1)} c_{i \alpha},
\end{gathered}
$$

respectively, where $\Pi_{\alpha \beta}=\sum_{i=0}^{8} N_{i}^{(0)} c_{i \alpha} c_{i \beta}$ is the momentum flux tensor. To recover the continuity equation (3) we, like Halliday et al. [1], choose the first-order source term to be

$$
S_{i}^{(1)}=-\frac{W_{i} \rho u_{y}}{y},
$$

where the weights $W_{i}, i=0, \ldots, 8$, are given by Eq. (12). Note that

$$
\begin{gathered}
\sum_{i=0}^{8} S_{i}^{(1)}=-\frac{\rho u_{y}}{y}, \\
\sum_{i=0}^{8} S_{i}^{(1)} c_{i \alpha}=0 .
\end{gathered}
$$

Our analysis now proceeds in the same fashion as that of Halliday et al. [1] but our second-order expression differs from previous derivations of axisymmetric LBM's in the following way. Halliday et al. [1] and Premnath and Abraham [2] borrow the $O\left(\epsilon^{2}\right)$ terms in the expansion of the evolution equation from the unadjusted LBGK equation to find at second order in $\epsilon$ 


$$
\partial_{t_{2}} N_{i}^{(0)}+\left(\partial_{t_{1}}+c_{i \alpha} \partial_{\alpha}\right)\left(1-\frac{\omega}{2}\right) N_{i}^{(1)}=-\omega N_{i}^{(2)}+S_{i}^{(2)} .
$$

We argue that the above equation is not correct, and thus neither is the expression for $S_{i}^{(2)}$ in [1], as we now demonstrate.

At order $\epsilon^{2}$ we obtain

$$
\begin{gathered}
\partial_{t_{2}} N_{i}^{(0)}+\partial_{t_{1}} N_{i}^{(1)}+c_{i \alpha} \partial_{\alpha} N_{i}^{(1)}+\frac{1}{2} \partial_{t_{1}} \partial_{t_{1}} N_{i}^{(0)}+c_{i \alpha} \partial_{t_{1}} \partial_{\alpha} N_{i}^{(0)} \\
+\frac{1}{2} c_{i \alpha} c_{i \beta} \partial_{\alpha} \partial_{\beta} N_{i}^{(0)}=-\omega N_{i}^{(2)}+S_{i}^{(2)} .
\end{gathered}
$$

If, using Eqs. (18) and (21), we write Eq. (25) in a similar form to Halliday et al. [1] and Premnath and Abraham [2], i.e.,

$$
\begin{aligned}
\partial_{t_{2}} N_{i}^{(0)} & +\left(\partial_{t_{1}}+c_{i \alpha} \partial_{\alpha}\right)\left(1-\frac{\omega}{2}\right) N_{i}^{(1)}-\left(\partial_{t_{1}}+c_{i \alpha} \partial_{\alpha}\right) \frac{W_{i} \rho u_{y}}{2 y} \\
= & -\omega N_{i}^{(2)}+S_{i}^{(2)},
\end{aligned}
$$

we see the presence of additional terms involving $S_{i}^{(1)}$, which are missing in Eq. (10) in [1]. More precisely, the coupling of the two source terms occurs through the nonequilibrium part of the distribution function $N_{i}^{(1)}$. Substituting Eq. (18) into Eq. (26) [or, alternatively, Eq. (25)] yields the following axisymmetric terms that are not present in the unadjusted LBGK equation:

$$
-\frac{1}{\omega}\left(\partial_{t_{1}}+c_{i \alpha} \partial_{\alpha}\right) \frac{W_{i} \rho u_{y}}{y}-S_{i}^{(2)}=\frac{1}{\omega}\left(\partial_{t_{1}}+c_{i \alpha} \partial_{\alpha}\right) S_{i}^{(1)}-S_{i}^{(2)} .
$$

Comparing this with the expression (17) in Halliday et al. [1], we see that our second-order axisymmetric contribution does not include the $-S_{i}^{(1)} / 2$ term. We suggest that (27) is the correct form for the expanded LBGK expression with an additional geometrical force term, and note that Eq. (26) is consistent with the generalized expansion of LBGK equations with additional forces, as described by Guo et al. [10].

Applying the mass conservation constraint to Eq. (25) gives

$$
\partial_{t_{2}} \rho+\frac{1}{2} \partial_{t_{1}} \partial_{t_{1}} \rho+\partial_{t_{1}} \partial_{\alpha} \rho u_{\alpha}+\frac{1}{2} \partial_{\alpha} \partial_{\beta} \Pi_{\alpha \beta}=\sum_{i=0}^{8} S_{i}^{(2)},
$$

and upon using Eqs. (19) and (20) we find that

$$
\partial_{t_{2}} \rho-\frac{1}{2} \partial_{t_{1}} \frac{\rho u_{y}}{y}=\sum_{1=0}^{8} S_{i}^{(2)} \text {. }
$$

Adding the above equation to Eq. (19) shows that

$$
\partial_{t} \rho+\partial_{\alpha} \rho u_{\alpha}=-\frac{\rho u_{y}}{y}+\frac{1}{2} \partial_{t_{1}} \frac{\rho u_{y}}{y}+\sum_{i=0}^{8} S_{i}^{(2)},
$$

so to recover the correct continuity equation we require

$$
\sum_{i=0}^{8} S_{i}^{(2)}=-\frac{1}{2 y} \partial_{t_{1}} \rho u_{y}=\frac{1}{2 y} \partial_{\alpha} \Pi_{\alpha y} .
$$

When the momentum conservation constraint is applied to Eq. (25) we find the following equality:

$$
\begin{gathered}
\partial_{t_{2}} \rho u_{\alpha}+\partial_{\beta} Q_{\alpha \beta}+\frac{1}{2} \partial_{t_{1}} \partial_{t_{1}} \rho u_{\alpha}+\partial_{t_{1}} \partial_{\beta} \Pi_{\alpha \beta}+\frac{1}{2} \partial_{\beta} \partial_{\gamma} P_{\alpha \beta \gamma} \\
=\sum_{i=0}^{8} S_{i}^{(2)} c_{i \alpha},
\end{gathered}
$$

where $Q_{\alpha \beta}=\sum_{i=0}^{8} N_{i}^{(1)} c_{i \alpha} c_{i \beta}$ and $P_{\alpha \beta \gamma}=\sum_{i=0}^{8} N_{i}^{(0)} c_{i \alpha} c_{i \beta} c_{i \gamma}$. This equation can be simplified using Eq. (20) to give

$$
\partial_{t_{2}} \rho u_{\alpha}+\partial_{\beta} Q_{\alpha \beta}+\frac{1}{2} \partial_{t_{1}} \partial_{\beta} \Pi_{\alpha \beta}+\frac{1}{2} \partial_{\beta} \partial_{\gamma} P_{\alpha \beta \gamma}=\sum_{i=0}^{8} S_{i}^{(2)} c_{i \alpha},
$$

where, from Eq. (18),

$$
Q_{\alpha \beta}=-\frac{1}{\omega}\left(\partial_{t_{1}} \Pi_{\alpha \beta}+\partial_{\gamma} P_{\alpha \beta \gamma}+\frac{\rho u_{y}}{3 y} \delta_{\alpha \beta}\right) .
$$

For a D2Q9 lattice the tensors $\boldsymbol{\Pi}$ and $\mathbf{P}$ are found to have the following form:

$$
\Pi_{\alpha \beta}=\frac{\rho}{3} \delta_{\alpha \beta}+\rho u_{\alpha} u_{\beta},
$$

$$
P_{\alpha \beta \gamma}=\frac{\rho u_{\alpha}}{3}\left(u_{\gamma} \delta_{\alpha \beta}+u_{\beta} \delta_{\alpha \gamma}+u_{\alpha} \delta_{\beta \gamma}\right)
$$

A little algebra now shows that Eq. (33) may be written as

$$
\begin{gathered}
\partial_{t_{2}} \rho u_{\alpha}-\nu\left(\partial_{\alpha} \partial_{\alpha} \rho u_{\alpha}+\partial_{\alpha} \partial_{\beta} \rho u_{\beta}+\partial_{\alpha} \frac{\rho u_{y}}{y}-2 \partial_{\alpha} \frac{\rho u_{y}}{y}\right) \\
-\frac{1}{3 \omega} \partial_{\alpha} \frac{\rho u_{y}}{y}=\sum_{i=0}^{8} S_{1}^{(2)} c_{i \alpha},
\end{gathered}
$$

where

$$
\nu=\frac{1}{3}\left(\frac{1}{\omega}-\frac{1}{2}\right)
$$

is the kinematic viscosity. If we assume the fluid is incompressible then Eq. (19) tells us that

$$
\partial_{\alpha} \partial_{\beta} \rho u_{\beta}+\partial_{\alpha} \frac{\rho u_{y}}{y}=0,
$$

which allows us to write Eq. (37) as

$$
\partial_{t_{2}} \rho u_{\alpha}-\nu \partial_{\alpha} \partial_{\alpha} \rho u_{\alpha}=\sum_{i=0}^{8} S_{i}^{(2)} c_{i \alpha}-2 \nu \partial_{\alpha} \frac{\rho u_{y}}{y}+\frac{1}{3 \omega} \partial_{\alpha} \frac{\rho u_{y}}{y} .
$$

Summing the first- and second-order momentum equations yields 


$$
\begin{aligned}
\partial_{t} \rho u_{\alpha}+\partial_{\beta} \Pi_{\alpha \beta}-\nu \partial_{\alpha} \partial_{\alpha} \rho u_{\alpha}= & \sum_{i=0}^{8} S_{i}^{(2)} c_{i \alpha}-2 \nu \partial_{\alpha} \frac{\rho u_{y}}{y} \\
& +\frac{1}{3 \omega} \partial_{\alpha} \frac{\rho u_{y}}{y}
\end{aligned}
$$

and since $\partial_{\beta} \Pi_{\alpha \beta}=\partial_{\alpha} \rho / 3+\rho u_{\alpha} \partial_{\alpha} u_{\beta}$ we find the momentum equation in vector form:

$$
\rho \frac{D \mathbf{u}}{D t}+\nabla P-\rho \nu \nabla^{2} \mathbf{u}=\sum_{i=0}^{8} S_{i}^{(2)} c_{i \alpha}-2 \nu \partial_{\alpha} \frac{\rho u_{y}}{y}+\frac{1}{3 \omega} \partial_{\alpha} \frac{\rho u_{y}}{y},
$$

where the left-hand side contains the terms in the standard Navier-Stokes equations and the terms on the right-hand side must deliver the extra axisymmetric contributions. Looking at the components of the above momentum equation and the second-order mass equation, we see that $S_{i}^{(2)}$ must conform to the following three conditions:

$$
\begin{gathered}
\sum_{i=0}^{8} S_{i}^{(2)}=\frac{1}{2 y} \partial_{\beta} \Pi_{y \beta}, \\
\sum_{i=0}^{8} S_{i}^{(2)} c_{i x}=\frac{\rho \nu}{y}\left(\partial_{y} u_{x}+\partial_{x} u_{y}\right)-\frac{\rho}{6 y} \partial_{x} u_{y}, \\
\sum_{i=0}^{8} S_{i}^{(2)} c_{i y}=\left(2 \nu-\frac{1}{6}\right) \frac{\rho \nu}{y}\left(\partial_{y} u_{y}-\frac{u_{y}}{y}\right) .
\end{gathered}
$$

To simplify the above relations and follow the lattice Boltzmann methodology as closely as possible, we try to relate $S_{i}^{(2)}$ to moments of the distribution function. Recalling that

$$
\begin{aligned}
Q_{\alpha \beta} & =-\frac{1}{\omega}\left(\partial_{t_{1}} \Pi_{\alpha \beta}+\partial_{\gamma} P_{\alpha \beta \gamma}+\frac{\rho u_{y}}{3 y} \delta_{\alpha \beta}\right) \\
& =-\frac{\rho}{3 \omega}\left(\partial_{\alpha} u_{\beta}+\partial_{\beta} u_{\alpha}+\frac{u_{y}}{y} \delta_{\alpha \beta}\right)
\end{aligned}
$$

enables us to find most of the required gradients in terms of the moments of $N_{i}^{(1)}$ :

$$
\frac{\nu \rho}{y}\left(\partial_{x} u_{y}+\partial_{y} u_{x}\right)=-\frac{6 \nu}{(6 \nu+1) y} \sum_{i=0}^{8} N_{i}^{(1)} c_{i x} c_{i y}
$$

$$
\begin{aligned}
\left(2 \nu-\frac{1}{6}\right) \frac{\rho \nu}{y}\left(\partial_{y} u_{y}-\frac{u_{y}}{y}\right) & \\
& =\frac{(1-12 \nu)}{y}\left(\frac{1}{2(1+6 \nu)} \sum_{i=0}^{8} N_{i}^{(1)} c_{i y}^{2}+\frac{\rho u_{y}}{4 y}\right) .
\end{aligned}
$$

A suitable choice of $S_{i}^{(2)}$ can now be found by inspection. Given below are our first- and second-order source terms that meet the required conditions (42)-(44) and therefore recover the axisymmetric Navier-Stokes equations in the macroscopic limit:

$$
\begin{gathered}
S_{i}^{(1)}=-\frac{W_{i} \rho u_{y}}{y} \\
S_{i}^{(2)}=\frac{3 W_{i}}{y}\left[\frac{\rho c_{i y}^{2}}{2 y}\left(u_{x} \partial_{x} u_{y}-\frac{3 u_{y} \omega}{2} Q_{x x}+\frac{\rho u_{y}^{2}}{2 y}-3 u_{y} \omega Q_{y y}-\frac{\rho u_{y}^{2}}{y}\right)\right. \\
-c_{i x}\left(\frac{6 \nu}{(6 \nu+1) y} Q_{x y}+\frac{1}{6 y} \partial_{x} u_{y}\right)+c_{i y}(1-12 \nu) \\
\left.\quad \times\left(\frac{1}{2(1+6 \nu)} Q_{y y}+\frac{\rho u_{y}}{4 y^{2}}\right)\right]
\end{gathered}
$$

The differences between our model and that of Halliday et al. [1] should now be highlighted. Our second-order source term is given mainly in terms of the tensor $\mathbf{Q}$, which is the third moment of the nonequilibrium distribution function $N_{i}^{(n e)}$. Therefore, we argue that this derivation is more sympathetic to the lattice Boltzmann philosophy. Another advantage of this approach is the reduction in the amount of numerical differentiation that needs to be performed compared to the lattice Boltzmann models of Halliday et al. [1] and Premnath and Abraham [2]. In the modified LBM described here, only one term, viz., $\partial_{x} u_{y}$, in the expression for $S_{i}^{(2)}$ needs to be approximated using finite differences compared with five terms in [1]. Although Haliday et al. [1] comment that components of the velocity gradient tensor can, in principle, be evaluated from appropriate higher-order moments of the nonequilibrium function, in practice we are of the opinion that it is not possible to express $\partial_{x} u_{y}$ in this manner. Numerical evidence for the improved efficiency of the axisymmetric LBE presented here will be given in a subsequent presentation. The application of the method to a number of benchmark problems will also be given there.

Finally, the analysis performed here is of the same form and performed to the same order as that presented in [1] but delivered in a different manner. We argue that this method is more transparent than that of Halliday et al. [1] and exposes an additional term in their second-order expansion of the evolution equation. The analysis performed in this paper is consistent with the general form of LBE equations with additional forcing given by Guo et al. [10].
[1] I. Halliday, L. A. Hammond, C. M. Care, K. Good, and A. Stevens, Phys. Rev. E 64, 011208 (2001).

[2] K. N. Premnath and J. Abraham, Phys. Rev. E 71, 056706 (2005).
[3] Y. Qian, D. d'Humières, and P. Lallemand, Europhys. Lett. 17, 479 (1992).

[4] F. Higuera, S. Succi, and R. Benzi, Europhys. Lett. 9, 345 (1989). 
[5] A. Ladd and R. Verberg, J. Stat. Phys. 104, 1191 (2001).

[6] D. Grunau, S. Chen, and K. Eggert, Phys. Fluids A 5, 2557 (1993).

[7] M. R. Swift, W. R. Osborn, and J. M. Yeomans, Phys. Rev. Lett. 75, 830 (1995).
[8] X. Shan and H. Chen, Phys. Rev. E 47, 1815 (1993).

[9] I. Ispolatov and M. Grant, Phys. Rev. E 65, 056704 (2002).

[10] Z. Guo, C. Zheng, and B. Shi, Phys. Rev. E 65, 046308 (2002). 